\title{
O DIZER DE FONOAUDIÓLOGOS SOBRE A EVOLUÇÃO TERAPÊUTICA DE CRIANÇAS COM DISTÚRBIOS FONOLÓGICOS/FONÉTICOS
}

\section{What speech therapist are telling about therapeutic evolution of children with phonological/phonetic disorders}

\author{
Ana Paula Ramos (1), Renata Reginato (2), Vanessa Panda Deuschle ${ }^{(3)}$
}

\begin{abstract}
RESUMO
Objetivos: investigar fatores que contribuem de forma decisiva na evolução terapêutica nos casos de distúrbios fonológico/fonético, bem como quais abordagens são utilizadas pelos fonoaudiólogos para tratar tais distúrbios. Métodos: dezoito fonoaudiólogos que atendem tal distúrbio foram entrevistados sobre questões relativas à evolução e métodos terapêuticos. Resultados: demonstram um grupo de profissionais provenientes de distintas instituições com predomínio do uso de abordagens fonológicas, embora citem a problemática emocional como um aspecto que dificulta a evolução terapêutica. Afirmam não terem formação para lidar com tal aspecto, mas não a buscam em supervisões ou cursos de formação. Conclusões: a dificuldade mais mencionada foi a resistência da criança a aceitar o trabalho instrumental de fala, sendo esta encarada como de origem emocional, sendo esse aspecto um dos referidos como deficitários na formação profissional. Abordagens fonéticas e fonológicas foram as mais citadas pelos profissionais.
\end{abstract}

DESCRITORES: Fonoterapia; Transtornos da Articulação; Fonética

\section{INTRODUÇÃO}

Define-se desvio fonológico como sendo uma dificuldade de fala, caracterizada pelo uso inadequado dos sons, de acordo com a idade e com variações regionais, que podem envolver erros na produção, percepção ou organização dos sons ${ }^{1-3}$. Há estudos que demonstram haver uma grande prevalência de alterações fonológicas ${ }^{4}$, em torno de $8,27 \%$ aos 7 anos de idade, o que demanda não só programa de intervenções terapêuticas efetivas, como também medidas preventivas.

Da década de 70 para até os dias atuais, ocorreu a mudança do foco terapêutico instrumental motor no fonema isolado, para o foco em aspectos

(1) Fonoaudióloga; Professora Doutora do Programa de PósGraduação em Distúrbios da Comunicação Humana da Universidade Federal de Santa Maria, UFSM, Santa Maria, RS; Doutora em Lingüística Aplicada pela Pontifícia Universidade Católica do Rio Grande do Sul.

(2) Fonoaudióloga; Clínica Renata Reginato.

(3) Fonoaudióloga; Prefeitura Municipal de São Francisco de Assis, RS; Mestranda em Distúrbios da Comunicação Humana pela Universidade Federal de Santa Maria. linguísticos. A noção de processo fonológico, de hierarquias de aquisição fonológica e a generalização estrutural na terapia são aspectos discutidos em muitos artigos da literatura tanto em relação aos distúrbios exclusivamente fonológicos como para os mistos (fonético-fonológicos) ${ }^{5-7}$.

Diante de uma séria de consequências comunicativas e escolares às quais os sujeitos com tais distúrbios se deparam, é imprescindível que essas crianças recebam ajuda de profissionais especializados para a obtenção de uma evolução terapêutica satisfatória ${ }^{8-12}$. Vários estudos têm abordado aspectos familiais, perceptivos e gerais do grupo desse grupo de crianças ${ }^{13-15}$, mas poucos tematizam as dificuldades terapêuticas advindas de cada abordagem teórica.

Há estudos que se referem a uma heterogeneidade nos casos. Existem casos mais demorados que demandam a necessidade de manejar aspectos que não os instrumentais, apontando dificuldades evolutivas na terapia ${ }^{16-18}$ e propondo novos fazeres, sobretudo centrado no lúdico ${ }^{19}$. Há, portanto, aspectos relevantes a serem investigados, que são os fatores que facilitam ou dificultam a aceitação da 
demanda pedagógica ("aprender os sons") do terapeuta pela criança, o que sugere a necessidade de investigação da evolução terapêutica de crianças com tais distúrbios na vivência clínica dos fonoaudiólogos. A partir dessa reflexão surgiu a proposta de investigar o discurso dos fonoaudiólogos a respeito.

Assim, esta pesquisa teve como objetivos principais investigar fatores que contribuem de forma decisiva na evolução terapêutica nos casos de distúrbios fonológico/fonético, bem como quais abordagens são utilizadas pelos fonoaudiólogos para tratar tais distúrbios.

\section{MÉTODOS}

A pesquisa realizada foi do tipo observacional, descritiva e transversal, sendo que a população da presente pesquisa foi composta por profissionais que atuam com crianças que apresentam desvio fonológico/distúrbio articulatório.
A amostra, de conveniência, constou de 18 fonoaudiólogas que atendessem desvio fonológico / distúrbio articulatório. As mesmas foram escolhidas por se tratarem de profissionais conhecidas nas áreas de linguagem oral e/ou motricidade orofacial no município de Porto Alegre e de alguns municípios vizinhos. Também foram priorizadas profissionais que ministrassem aulas na área de fala nos cursos de graduação em Porto Alegre e região metropolitana.

A coleta foi realizada através do contato com as fonoaudiólogas, que esclarecidas sobre o teor da pesquisa, receberam o termo de consentimento livre e esclarecido para assinar, caso concordassem em participar. Tiveram assegurado assim os direitos voluntariado e confidencialidade de seus dados pessoais.

Após a assinatura do termo, procedeu-se a entrevista das profissionais sobre sua experiência com casos de desvio fonológico/distúrbio articulatório. A mesma foi registrada através de gravador Sony, em fitas cassetes Philips, para posterior transcrição.

\section{QUESTIONÁRIO SOBRE "O DIZER DE FONOAUDIÓLOGOS SOBRE A EVOLUÇÃO TERAPÊUTICA DE CRIANÇAS COM DESVIOS FONOLÓGICOS EVOLUTIVOS/DISTÚRBIOS ARTICULATÓRIOS”}

\section{- DAdOS IDENTIFICAÇÃo}

Nome:

Ano de Graduação:

Instituição onde se graduou:

Pós-Graduação:

Curso: Instituição:

Atividade Profissional:

Ano de Conclusão:

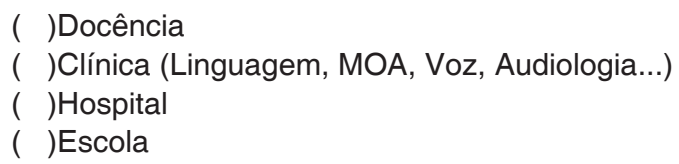

\section{- ENTREVISTA}

1. Qual a abordagem teórica que você utiliza para realizar a terapia dos Distúrbios Articulatórios/desvios fonológicos?

2. Onde recebeu esta formação? Na graduação, pós-graduação, autodidata?

3. Sintetize os procedimentos que você utiliza para avaliação?

4. Você acha esses casos de fácil evolução ou não? Dê exemplos dos dois extremos ou de diferentes evoluções?

5. Que fatores você identifica como intervenientes na evolução?

6. Você acha que a sua formação permite pensar bem esses fatores?

7. Diga o que conhece de cada tipo de abordagem teórica: motricidade oral, linguística (fonologia), interacionismo.

8. Você identifica fases na terapia? Quais?

9. Você tem por costume trabalhar esses pacientes em conjunto com outro profissional como, por exemplo, da psicologia? Como faz o encaminhamento?

10. Você usa o lúdico na terapia? Como? Dê exemplos. 
A entrevista constou de dados de identificação, formação, experiência profissional e questões abertas sobre opiniões acerca dos desvios fonológicos/distúrbios articulatórios quanto à evolução terapêutica, fatores que facilitam ou dificultam essa evolução e abordagens teórica e prática desses distúrbios. A entrevista foi realizada no local de escolha da profissional, o consultório ou em instituições de ensino superior onde trabalhavam. A duração média da entrevista foi de 40 minutos.

O entrevistador procurou não intervir para que o relato fluísse, apenas fazendo esclarecimentos quando necessários sobre as questões formuladas (Figura 1).

Esta pesquisa se insere no projeto "Desvio Fonológico: caracterização, avaliação e terapia", aprovado no Comitê de Ética em Pesquisa da Universidade Luterana do Brasil sob o protocolo 107/2002.

Após a transcrição das fitas, as respostas foram analisadas qualitativamente através da criação de categorias para posterior classificação individual e em grupos. Também foi feita a análise da frequência dessas categorias na amostra. A seguir, os resultados foram confrontados com a bibliografia a respeito desses distúrbios.

\section{RESULTADOS}

Dos 18 profissionais entrevistados, cinco $(28 \%)$ apresentavam menos de cinco anos de atuação profissional, nove (50\%) possuem de cinco a 12 anos e quatro $(22 \%)$ tinham período de atuação profissional superior a 12 anos. Desta maneira, observa-se que a maioria dos profissionais entrevistados tem de cinco a doze anos de formação, conforme representa a Tabela 1.

Tabela 1 - Tempo de graduação dos profissionais entrevistados

\begin{tabular}{cc}
\hline Tempo de Graduação & Número de profissionais \\
\hline Superior a 12 anos & 4 \\
Entre 5 e 12 anos & 9 \\
Inferior a 5 anos & 5 \\
\hline
\end{tabular}

A Tabela 2 demonstra que a amostra se constituiu de profissionais provenientes de diferentes instituições, sendo mais frequentes as três instituições que possuem cursos de Fonoaudiologia há mais tempo no Estado do Rio Grande do Sul.
Tabela 2 - Distribuição percentual por instituição de graduação

\begin{tabular}{cc}
\hline Instituição de Graduação & \% de profissionais \\
\hline A & 39 \\
B & 17 \\
C & 33 \\
D & 6 \\
E & 6 \\
\hline
\end{tabular}

Pode-se observar que a maioria dos fonoaudiólogos entrevistados frequentou mestrado ou especialização, sendo que sete (39\%) desses profissionais realizaram mestrado, e seis especialização, conforme a Tabela 3.

Tabela 3 - Formação em pós-graduação

\begin{tabular}{cc}
\hline Curso Pós-graduação & Número de profissionais \\
\hline Especialização e Mestrado & 2 \\
Mestrado & 7 \\
Especialização & 6 \\
Nenhum & 3 \\
\hline
\end{tabular}

Todos os profissionais clinicam, conforme indicado na Tabela 4 , sendo que oito $(44,4 \%)$ também exercem a docência em ensino superior.

\section{Tabela 4 - Atuação profissional}

\begin{tabular}{cc}
\hline Tipo de atuação & Número de profissionais \\
\hline Docência & 8 \\
Clínica & 18 \\
Hospital & 2 \\
Escola & 4 \\
\hline
\end{tabular}

Ao serem questionados sobre onde receberam a formação para atuar com esses distúrbios, 15 $(92,22 \%)$ elegeram a graduação, sete $(39 \%)$ a pósgraduação e sete $(39 \%)$ referiram ser autodidatas, demonstrando a importância da academia formal e do estudo individual para a formação profissional. Poucos citaram as supervisões e cursos como sendo lugares importantes para a formação, conforme demonstrado na Figura 2.

Observa-se na Tabela 5 que os procedimentos mais utilizados para avaliação são os testes, dos quais 15 (92,2\%) com base fonológica e 10 (55,5\%) outros tipos de avaliação. Poucos citaram a entre- 


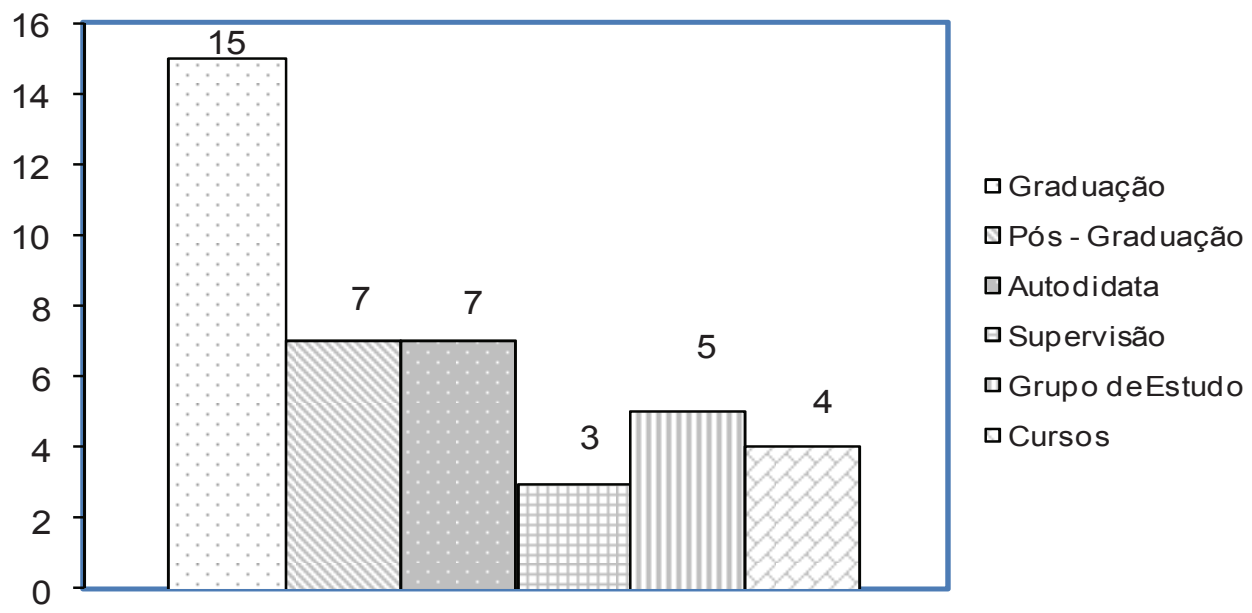

Figura 2 - "Onde recebeu a formação para atender este distúrbio?”

vista, embora se imagine que sempre a utilizem, parecendo assim ser algo à parte da avaliação. A investigação da relação da criança com a família foi pouco comentada.

\section{Tabela 5 - Procedimentos utilizados para avaliação}

\begin{tabular}{lc}
\hline Procedimentos para Avaliação & $\begin{array}{c}\text { Número de } \\
\text { Profissionais }\end{array}$ \\
\hline $\begin{array}{l}\text { Testes Formais ou Instrumentos } \\
\text { de Coleta }\end{array}$ & 24 \\
$\begin{array}{l}\text { Atividades que envolvem Fala } \\
\text { Espontânea }\end{array}$ & 13 \\
$\begin{array}{l}\text { Teste de Habilidade do } \\
\text { Processamento Auditivo }\end{array}$ & 5 \\
$\begin{array}{l}\text { Entrevista } \\
\text { Observação do relacionamento } \\
\text { com a mãe }\end{array}$ & 5 \\
$\begin{array}{l}\text { Exames Complementares } \\
\text { Brinquedo } \\
\text { Motricidade Oral }\end{array}$ & 2 \\
\hline
\end{tabular}

* Respostas Múltiplas

Ao serem questionados sobre a facilidade ou dificuldade de evolução desses casos, 13 (72,2\%) fonoaudiólogos responderam que depende do caso, podendo ser fácil ou difícil, e cinco (28\%) que são casos de fácil evolução.

Conforme apresentado na categorização da Tabela 6, observa-se uma frequência alta de fatores ligados à esfera emocional (EE), perfazendo um total de vinte e cinco menções, sejam elas relacionadas à criança, à família ou ao vínculo com o terapeuta. Em segundo lugar, aparecem as alterações de habilidades auditivas, memória, etc. $(\mathrm{AH})$, com
14 menções. Apenas em uma resposta aparece a característica linguística (CL).

Observa-se na Tabela 6 que as respostas indicam um trabalho mais próximo à abordagem interacionista, visto que os profissionais destacaram mais os aspectos da esfera emocional, contradizendo a Tabela 5 que indicava um trabalho mais organicista ou cognitivista, no qual há uma maior valorização das habilidades do sujeito. Portanto, vê-se uma noção de interacionismo diferente, no discurso do grupo aqui entrevistado, que se relaciona mais à ação/interação com o outro.

De acordo com a categorização apresentada na Tabela 7, observa-se que os fatores aos quais os profissionais relacionaram como sendo intervenientes na evolução terapêutica foram, em sua maioria, os relacionados ao sujeito (S), após os referentes ao terapeuta $(\mathrm{T})$ e apenas um relacionou à família ou ao ambiente (FA).

Os fatores intervenientes citados, relacionados com o sujeito, combinam com a esfera emocional, porém a relação com a família ou ambiente foi pouco indicada.

Outros dados da entrevista demonstram 15 $(83 \%)$ dos profissionais acreditam que sua formação faz com que seja possível pensar bem esses fatores, enquanto dois $(11 \%)$ referiram que não. Além disso, demonstra que todos os profissionais utilizam o lúdico na terapia, mas somente dois fonoaudiólogos usam o lúdico com os conceitos terapêuticos da psicologia, como, por exemplo, o uso do brinquedo livre.

A maioria dos profissionais entrevistados relatou trabalhar em paralelo com outros profissionais, tais como psicólogos, otorrinolaringologistas, psicopedagogos, ortodontistas, pediatras, neurologistas, fisioterapeutas ou terapeutas ocupacionais. 
Tabela 6 - Categorização de respostas à pergunta "Você acha esses casos de fácil evolução ou não? Por quê?"

\begin{tabular}{ccc}
\hline Categorização & Depende do Caso & No de Menções \\
\hline & Fácil: & $\mathbf{1 3}$ \\
AH ou EE & desvio fonológico puro & 4 \\
EE & família cooperativa & 5 \\
EE & paciente disciplinado ou cooperativo & 4 \\
EE & motivação para a mudança & 1 \\
EE & Vínculo & 1 \\
& Difícil: & \\
EE & componente emocional & 6 \\
AH & alteração motora & 6 \\
AH & processamento auditivo central & 1 \\
CL & Fácil & 1 \\
\hline & frocessos atípicos e quantidade de processos fonológicos & $\mathbf{5}$ \\
\hline EE & sem comprometimento motor & 1 \\
AH & sem comprometimento emocional & 2 \\
EE & sem problemas associados & 2 \\
AH ou EE & EE & 1 \\
TOTAL & AH & $\mathbf{2 5}$ \\
& CL & $\mathbf{1 4}$ \\
& & $\mathbf{1}$
\end{tabular}

* Respostas Múltiplas

Tabela 7 - Fatores intervenientes na evolução terapêutica

\begin{tabular}{|c|c|c|}
\hline Categorização & Fatores intervenientes na Evolução & $\begin{array}{l}\text { Número de } \\
\text { Profissionais }\end{array}$ \\
\hline S & Emocional & 18 \\
\hline $\mathrm{S}$ & motricidade oral & 3 \\
\hline$S$ & problemas neurológicos & 1 \\
\hline $\mathrm{S}$ & déficit de memória e atenção & 2 \\
\hline S & motivação do paciente & 2 \\
\hline S & fatores genéticos & 1 \\
\hline $\mathrm{S}$ & criança não cooperativa & 1 \\
\hline Te $S$ & respeitar os limites do paciente & 1 \\
\hline $\mathrm{T}$ & Vínculo & 3 \\
\hline $\mathrm{T}$ & técnicas utilizadas & 2 \\
\hline $\mathrm{T}$ & enxergar a criança como um todo & 1 \\
\hline $\mathrm{T}$ & avaliação auditiva & 1 \\
\hline $\mathrm{T}$ & avaliação e diagnóstico adequado & 1 \\
\hline $\mathrm{T}$ & Reavaliações & 1 \\
\hline $\mathrm{T}$ & frequência do trabalho & 1 \\
\hline FA & nível de estimulação linguística & 1 \\
\hline
\end{tabular}

* Respostas Múltiplas 
Para ilustrar podem-se citar as respostas de alguns fonoaudiólogos.

F1: “... sempre que eu detecto a necessidade eu procuro... e isso pode ser não só na área da psicologia, pode ser o psicopedagogo, pode ser em outras áreas".

F10: “... não abro mão de fazer o encaminhamento, de discutir o caso com o psicólogo, com o neurologista, com o pediatra, eu acho fundamental..."

F14: "Sim... tem uns que vem da psicóloga e têm... muitos eu encaminho. Tem também o ortodontista, o otorrinolaringologista... eu peço".

F17: "Cada paciente é um caso diferente, existe pacientes que precisam de um acompanhamento também psicológico e outros não, às vezes os pacientes têm atendimento psicopedagógico, às vezes o atendimento é fonoaudiológico junto com a terapia ocupacional ou com a fisioterapia, sei lá, depende do paciente."

Conforme representado na Tabela 8, observa-se que vários fonoaudiólogos optam por mais de uma abordagem teórica.

\section{Tabela 8 - "Qual a abordagem teórica que você utiliza?"}

\begin{tabular}{lc}
\hline Abordagem teórica & Número de Profissionais \\
\hline Fonologia & 16 \\
Interacionismo como & 13 \\
Ação/relação & 18 \\
Motricidade Oral & 05 \\
Interacionismo brasileiro & 01 \\
Não Respondeu & \\
Totais & $\mathbf{0 9}$ \\
$\quad \mathbf{0 1}$ abordagem & $\mathbf{0 6}$ \\
$\mathbf{0 2}$ abordagens & $\mathbf{0 2}$ \\
$\mathbf{0 3}$ abordagens & \\
\hline
\end{tabular}

* Respostas Múltiplas

Todos os fonoaudiólogos entrevistados demonstram conhecimento de motricidade orofacial, embora nem todos optem por abordagem com enfoque principal nesta linha. Dezesseis profissionais conhecem a abordagem fonológica, embora nem todos a elejam.

Quanto ao interacionismo, cinco fonoaudiólogos têm uma aproximação do conceito desta abordagem, e 13 relacionam interacionismo à ação/relação com paciente.

O profissional F15 demonstra bem, em sua fala, a distinção entre o interacionismo como sendo ação/relação com o paciente e a proposta teórica de alguns trabalhos.
F15 - "Quanto à abordagem, paradigma teórico do interacionismo, eu acho que me falta conteúdo. Sei princípios básicos, mas não me aprofundei nisso, até em função de vir da linguística, onde o paradigma é a abordagem linguística, que é diferente do paradigma interacionista, mas eu uso a palavra interação de uma maneira mais... a atividade interação numa maneira mais flexível, menos comprometida com essa prisão à nomenclatura que eu sei que existe. Se tu usas a palavra interacionismo tu és chicoteado, então, assim, independente de paradigmas, a gente em consultório usa o tempo todo, não vive sem interação. Isso é coisa que a gente aprende."

Observa-se, portanto, até um receio de utilizar a terminologia relacionada ao interacionismo pela reação violenta que os profissionais, que se dizem adeptos dessa concepção, apresentam em caso de uso inadequado.

\section{DISCUSSÃO}

Em relação à entrevista como forma de inclusão da família na terapia, a falta de valorização da entrevista parece relacionar-se com déficits na formação em conteúdos teóricos e práticos mais direcionados para a área de entrevistas, e do uso da entrevista como algo sistemático na terapêutica infantil ${ }^{17}$. Acredita-se que o crucial é a inclusão da família no processo, e a própria tentativa de utilizar a técnica pode ser o único meio de conscientizar a família a buscar a ajuda e orientação necessárias ${ }^{16}$. Além do que, tendo um controle sobre a história do transtorno de fala e linguagem na família, pode-se saber se a criança tem possibilidade de ter ou não transtorno fonológico e quais processos ela tem tendência a usar ${ }^{15}$.

As habilidades do sujeito foram mais destacadas, dando assim a idéia de uma visão organicista ou cognitivista ${ }^{1-15}$ nas quais os dons e aptidões dos sujeitos são fundamentais para o desenvolvimento articulatório. Tal visão se diferencia de propostas interacionistas de linguagem, que se preocupam com a interação dialógica como foco no estudo da aquisição da linguagem típica e atípica e com o brincar como instrumental de intervenção ${ }^{16-19}$.

Talvez se justifique o fato dos testes serem os mais citados pelos profissionais entrevistados, por encontrar-se na literatura uma maior valorização destes, demonstrando a importância da realização de uma avaliação completa para que seja possível a determinação do sistema fonológico da criança e estabelecidas as metas e os objetivos da terapia ${ }^{3,10}$.

Embora um número menor de profissionais tenha referido a fala espontânea, acredita-se que a 
melhora desta seja utilizada como critério de avaliação para alta fonoaudiológica ${ }^{17}$.

A fala espontânea é um excelente meio para obtenção de uma amostra linguística, pois além de apresentar o sistema próprio da criança sem a possibilidade de desvirtuamento da imitação, mostra o fluxo da linguagem, isto é, a produção de fonemas, sílabas e palavras dentro de frases, propiciando a verificação das possíveis relações fonológicas entre palavras ${ }^{1,2,5}$.

Cabe ressaltar ainda, que o interacionismo, mencionado pelos fonoaudiólogos entrevistados, difere do interacionismo brasileiro, visto que este parte da concepção teórica de linguagem com duas faces, atividade e objeto. A criança, a princípio, não opera com categorias linguísticas determinadas. É através dos processos dialógicos há uma sistematização gradual de várias faces da linguagem e, dessa forma, os papéis sociais, inscritos em cada fragmento do discurso, são gradualmente assumidos e organizados pela criança ${ }^{18}$. Desse processo surge a possibilidade da criança se conceber, a si e ao outro, como sujeitos. O interacionismo adquire um sentido particular, o de "interação/relação" da criança com a língua/fala ${ }^{16,17}$.

Observa-se que, portanto, em comparação com a avaliação na qual testes com critérios linguísticos, como o instrumento de avaliação fonológica da criança ${ }^{10}$, são mais valorizados, surge apenas uma resposta colocando o locus da dificuldade evolutiva na característica linguística.

Ressaltando fatores da esfera emocional, é de extrema importância a participação familiar e a relação terapeuta-paciente no processo terapêutico, ressaltando a necessidade da conscientização e motivação do paciente sobre as suas necessida$\operatorname{des}^{8,17}$.

Essa questão, ao analisar a evolução dos casos de desvio fonológico, que dependeram de fatores como a valorização enquanto interlocutor no ambiente familiar e terapêutico, e resoluções da esfera emocional ${ }^{16-18}$.

Também, polemiza-se esta questão ao afirmar que, quando se fala do paciente, vem a questão do sujeito psicológico, ou seja, de condições intelectuais e emocionais como fatores que intervêm no processo terapêutico ${ }^{16}$. Tais fatores caminham ao lado da fala e da reflexão dos pesquisadores, são acessórios. Acessórios porque marginalizados na reflexão, mas, paradoxalmente, extremamente importantes já que determinantes da evolução e do sucesso do tratamento.

É fundamental a observação dos aspectos emocionais, cognitivos e sociais que envolvem a criança durante o processo terapêutico ${ }^{13,14}$. O terapeuta não deve descuidar desses fatores, que são indis- pensáveis para o bom andamento da dinâmica terapêutica. É importante a participação familiar para a motivação da criança no trabalho desenvolvido, como também no acompanhamento fora da sala de terapia, uma vez que o ser humano constrói-se em relação dialógica com as condições socioculturais de seu tempo.

Embora a maioria dos profissionais relate que sua formação permite pensar bem os fatores relacionados a esses distúrbios, observa-se uma carência de valorização teórica da entrevista e da avaliação da família durante o processo de avaliação.

Apesar de todos os fonoaudiólogos utilizarem o lúdico, apenas dois parecem ter um embasamento teórico para este uso, conforme já assinalaram alguns trabalhos sobre o a atividade lúdica de fonoaudiólogos ${ }^{19}$. Alguns pontos surgem para reflexão quanto a este receio ou mesmo insegurança manifesta na fala de F15.

O primeiro é que talvez haja uma divulgação e socialização inadequada da teoria interacionista já que há mais equívocos do que acertos na compreensão das bases teóricas e como se acredita que os profissionais, em geral, por acessarem outras teorias abstratas como o gerativismo, podem acessála em sua abstração.

O segundo ponto é que a teoria interacionista ainda não padronizou procedimentos em termos clínicos. Em geral, há muitos estudos críticos sobre a atuação fonoaudiológica behaviorista, mas não há oferta suficiente de uma padronização de ações com base interacionista, ou seja, procedimentos clínicos pensados, testados e divulgados de modo a facilitar a aplicação.

Em último lugar, pode-se hipotetizar que a educação de concepção mais behaviorista, à qual os próprios terapeutas são submetidos, cria um viés constante que, na hora da prática, leva-os a buscar modelos aplicativos também behavioristas.

Portanto, parece haver uma confluência de fatores que determinam a prática fonoaudiológica com esses distúrbios: concepções teóricas pouco conhecidas em sua radicalidade, padronização de procedimentos de avaliação realizados no gerativismo o que leva à utilização dessa teoria para a avaliação, uso de modelos fonológicos com base de raciocínio hierárquico no gerativismo, mas com concepção de relação e aprendizagem behavioristas, associados a uma sensibilidade para aspectos emocionais, embora sem formalização teórica desta.

Nota-se, portanto, a necessidade de uma maior reflexão sobre a compatibilidade entre os procedimentos de avaliação e os de terapia. De modo especial, há a necessidade de pensar sobre qual(ais) aporte(se) teórico(s) a Fonoaudiologia poderia utilizar para abordar questões da esfera emocional 
relacionadas a aspectos estruturais do sujeito e de seu dinâmica familiar.

Nas entrevistas foi possível observar profissionais que possuem a consciência dessa polêmica, como $\mathrm{F} 15$, e outros que possuem mais teorização sobre questões emocionais, outros sobre o brinquedo. Na verdade, a influência de sua formação da graduação e do contato profissional por ocasião de sua prática clínica parecem ser fatores determinantes no seu conhecimento e sensibilidade terapêutica, como já era esperado. Assim, cabe uma reflexão profunda, sobretudo nos cursos de graduação, sobre a formação eclética e a possibilidade de esta não estar sendo efetiva na teorização da prática. Isso não elimina a possibilidade saudável de uma formação com mais de uma concepção teórica.

Crê-se que, na medida em que a teoria interacionista gerar procedimentos clínicos, haja uma procura e entendimento maior dessa e isso faça com que haja uma opção maior pela mesma já que preconiza a inclusão da família e dos aspectos subjetivos na terapêutica, demanda esta observada nas entrevistas desta pesquisa.

\section{CONCLUSÃO}

Após a realização desta pesquisa é possível concluir que os fatores decisivos na evolução terapêutica são os emocionais e as alterações de motricidade oral, na visão do grupo investigado.

Em relação às dificuldades ou mesmo facilidades evolutivas, correlacionam as mesmas com esses aspectos, ou seja, se há maior dificuldade emocional e/ou orofacial, ocorre uma lentidão evolutiva maior.

Considerando as abordagens terapêuticas, as mais citadas são as fonológicas e as relacionadas à área da motricidade oral. Entre os princípios e estratégias mais utilizadas estão as fonéticas e as fonológicas, como por exemplo, a utilização do modelo de ciclos na terapêutica.

A maior parte dos profissionais afirmou que sua formação, acadêmica ou não, dá conta do trabaIho com a criança e família quanto aos aspectos mencionados como difíceis, sobretudo a resistência da criança a entrar no trabalho instrumental. No entanto, apenas um fonoaudiólogo afirmou a necessidade de aprimorar a formação na área psicológica ou psicanalítica.

\begin{abstract}
Purpose: to investigate relevant therapy factors on evolution of children with phonological/phonetic disorders and theatrical approaches of therapy chosen by therapists. Methods: eighteen speech therapists had been interviewed about therapeutic approaches and evolution of phonological/phonetic disorders children. Results: it shows that phonological and phonetical approaches are predominant despite emotional factors are relevant because they create child resistances to therapy. The therapists do not study these factors in supervision or graduation courses. Conclusions: the most mentioned difficulty was the child's resistance to accept the instrumental work of speech, and it is treated as an emotional factor, being this aspect one of those referred to as deficient in the professional qualification. Phonetic and phonological approaches are the more used in therapy.
\end{abstract}

KEYWORDS: Speech Therapy; Articulation Disorders; Phonetics

\section{REFERÊNCIAS}

1. Wertzner HB. Fonologia: desenvolvimento e alterações. In: Ferreira LP, Befi-Lopes DM, Limongi SCO. Tratado de fonoaudiologia. São Paulo: Roca; 2004. p. 772-86.

2. Mota HB. Fonologia: intervenção. In: Ferreira LP, Befi-Lopes DM, Limongi SCO. Tratado de fonoaudiologia. São Paulo: Roca; 2004. p.787-814. 3. Wertzner HF, Papp ACCS, Gálea DE dos S. Provas de nomeação e imitação como instrumentos de diagnóstico do transtorno fonológico. Pró-Fono. 2006; 18(3):303-12.
4. Patah LK, Takiuchi N. Prevalência das alterações fonológicas e uso de processos fonológicos em escolares aos 7 anos. Rev CEFAC. 2008; 10(2):158-67.

5. Ceron MI, Keske-Soares M. Terapia fonológica e a generalização a itens não utilizados no tratamento (outras palavras). Rev CEFAC. 2007; 9(4):453-60.

6. Pagliarin KC, Keske-Soares M. Abordagem contrastiva na terapia de desvios fonológicos: considerações teóricas. Rev CEFAC. 2007; 9(3):330-8. 
7. Rodríguez VMA, Leon SL. Contributions to the problems of generalization in the phonological intervention: a clinical approach. Rev CEFAC. 2007; 9(1):21-31.

8. Silva KC, Ramos APF, Wippel MLM. O modelo de oposições máximas como instrumento terapêutico em três casos de desvios fonológicos. J Bras Fonoaudiol. 2002; 3(13):292-7.

9. Rvachew S, Nowak M, Cloutier G. Effect of phonemic perception training on the speech production and phonological awareness skills of children with expressive phonological delay. Am J Speech Lang Pathol. 2004;13(3):250-63.

10. Yavas M, Hernandorena CLM, Lamprecht RR. Avaliação fonológica da criança: reeducação e terapia. Porto alegre: Artes Médicas; 1991. 148p.

11. Goorhuis-Brouwer SM, Knijff WA. Language disorders in young children: when is speech therapy recommended? Int J Pediatr Otorhinolaryngol. 2003; 67(5):525-9.

12. Glykas M, Chytas P. Technology assisted speech and language therapy. Int J Med Inform. 2004; 73(6):529-41.
13. Gillon GT. Facilitating phoneme awareness development in 3- and 4-year-old children with speech impairment. Lang Speech Hear Serv Sch. 2005; 36(4):308-24.

14. Broomfield J, Dodd B. Children with speech and language disability: caseload characteristics. Int $\mathrm{J}$ Lang Commun Disord. 2004; 39(3):303-24.

15. Wertzner HF, Papp ACCS. O aspecto familial e o transtorno fonológico. Pró-Fono. 2006; 18(2):151-60.

16. Benine R. "Ómideio!" O que é isto?: questões e reflexõess sobre dislalias, distúrbios funcionais e desvios fonológicos [tese]. São Paulo (SP): Pontifícia Universidade Católica; 2001.

17. Nobre TL, De-Vitto LPM. Desvios fonológicos: considerações bibliográficas relevantes sobre a avaliação e intervenção fonoaudiológica. J Bras Fonoaudiol. 2005; 5(23):424-30.

18. Panhoca I, Penteado RZ. Grupo terapêuticofonoaudiológico: a construção (conjunta) da linguagem e da subjetividade. Pró-Fono. 2006; 15(3):259-66.

19. Graña CG, Ramos AP. Falando com brinquedos: fazeres do fonoaudiólogo na atividade clínica com crianças. Letras UFRGS. 2006; 20(40/41):141-56.

DOI: 10.1590 / S1516-18462008005000010

RECEBIDO EM: 17/09/2007

ACEITO EM: 30/05/2008

Endereço para correspondência:

Ana Paula Ramos

Rua Raposo Tavares, 134/401

Santa Maria - RS

CEP: 97015-560

E-mail: ramos1964@uol.com.br 